\title{
The Hong Kong Reference Frameworks-for our doctors and our community
}

Monica MH Wong * , FHKAM (Community Medicine)

Primary Care Office, Department of Health, Hong Kong

Hong Kong Med J 2017;23:220-1

DOI: $10.12809 / \mathrm{hkmj} 175067$

\section{Introduction}

I remember that as a student, when I wanted to find papers about new developments in disease management, I needed to consult what looked like a set of encyclopaedias-the MEDLINE / Index Medicus. There followed a search in the medical library for hard copies of overseas medical journals. What a tedious job it was! Thanks to the invention of computers and evolvement of this cyber world, I can now sit comfortably anywhere and access all sorts of updated guidelines and recommendations with one click. Wait a minute, there are so many international guidelines available for any particular subject! I have been asking myself, "How best can we choose which one is most suitable for patients in Hong Kong? Can we have local protocols that address the needs of Hong Kong?"

\section{The Hong Kong Reference Frameworks}

The answer lies in a series of Hong Kong Reference Frameworks, or RFs for short, released after 2010 to cover the preventive care of children and older adults, as well as the prevention and treatment of two common chronic conditions (diabetes and hypertension) in the primary care setting. ${ }^{1-4}$ The development of RFs was one of the initiatives recommended by the then Working Group on Primary Care, chaired by the Secretary for Food and Health, and produced by the Task Force on Conceptual Model and Preventive Protocols under this Working Group. These frameworks are based on evidence from international literature with input from primary care physicians, as well as specialists from relevant Colleges of the Hong Kong Academy of Medicine, academic experts from the universities, doctors from the public and private sectors, and representatives from professional associations and patient groups.

It is hoped that these RFs will serve as a handy local source of information about evidence-based practice and a reference for doctors in their day-today work in a primary care setting.

\section{Our platform}

Being mindful of environmental conservation, these
RFs are available primarily on a web-based platform, on the website of the Primary Care Office at $<w w w$. pco.gov.hk>. Various aides mémoire are available to provide doctors with a quick reference during patient consultations. For example, A4 size cards that summarise the major recommendations, and cue cards for immunisation and health assessment. We also have a mobile application 'Framework@PC' that can be accessed from mobile devices to enable prompt retrieval of the major recommendations in the RFs.

You may ask, how have we 'advertised' these RFs to end users? How can we encourage more primary care doctors to adopt these RFs?

There is no surprise or magic answer. We have used various CME platforms. We are co-organising CME seminars with professional organisations (such as the Hong Kong Medical Association and Hong Kong Doctors Union). We run training programmes in collaboration with the Hong Kong College of Family Physicians to elaborate on the contents of the RFs. We have also submitted articles to the Hong Kong Medical Journal ${ }^{5-8}$ and other journals ${ }^{9}$ to facilitate a broad readership, at any time and in any place. This also enables RF-related articles to be indexed and searched on online medical publication databases, and hence further broadens our readership. Doctors listed in the Primary Care Directory will also be notified of any release of new modules and major updates of the existing RFs.

We believe that increasing the understanding of the general public about prevention and management of diseases is an effective means to create an environment that is conducive for doctors to implement RF recommendations for their patients. Over the years, we have produced information booklets or leaflets for the general public that will empower them by providing information about the recommendations of the RFs.

Although online viewing of the RFs does not necessarily imply a reader's adoption of the recommendations, viewing figures nevertheless provide an indication of readers' awareness of these documents. Since their publication on our website, we have recorded over 600000 downloads of the core documents and the various modules under the four RFs, with a consistent number of downloads every month. We know promulgation activities must 
continue so that these RFs reach as many doctors as possible. We will not stop.

\section{Future development}

New modules will continue to be produced, particularly for RFs relative to specific population groups, such as the module on development in children and another on cognitive impairment in older adults. Hopefully, the in-depth discussions and problem-solving algorithms in the modules will help professionals manage such issues in a primary care setting.

May I take this opportunity to call upon all doctors to support enhancement of primary care through the provision of high quality care that is comprehensive, continuing, co-ordinated, and person-centred. Although there are many ways to achieve this, applying the recommendations of the RFs in your patient care is certainly one of your best options. The contribution of each individual doctor contributes to the overall health of Hong Kong people. I know I can count on you.

\section{References}

1. Hong Kong Reference Framework for Preventive Care for Children in Primary Care Settings. Available from: http:// www.pco.gov.hk/english/resource/files/ref_framework_ children.pdf. Accessed 26 Apr 2017.

2. Hong Kong Reference Framework for Preventive Care for Older Adults in Primary Care Settings. Available from: http://www.pco.gov.hk/english/resource/files/ref framework_adults.pdf. Accessed 26 Apr 2017.

3. Hong Kong Reference Framework for Diabetes Care for Adults in Primary Care Settings. Available from: http:// www.pco.gov.hk/english/resource/files/RF_DM_full.pdf. Accessed 26 Apr 2017.

4. Hong Kong Reference Framework for Hypertension Care for Adults in Primary Care Settings. Available from: http:// www.pco.gov.hk/english/resource/files/RF_HT_full.pdf. Accessed 26 Apr 2017.

5. Wong MC, Sin CK, Lee JP. The reference framework for diabetes care in primary care settings. Hong Kong Med J 2012;18:238-46.

6. Griffiths SM, Lee JP. Developing primary care in Hong Kong: evidence into practice and the development of reference frameworks. Hong Kong Med J 2012;18:429-34.

7. Siu NP, Too LC, Tsang CS, Young BW. Translating evidence into practice: Hong Kong Reference Framework for Preventive Care for Children in Primary Care Settings. Hong Kong Med J 2015;21:261-8.

8. Sin CK, Fu SN, Tsang CS, Tsui WW, Chan FH. Prevention in primary care is better than cure: The Hong Kong Reference Framework for Preventive Care for Older Adults-translating evidence into practice. Hong Kong Med J 2015;21:353-9.

9. Cindy LK Lam, KH Ngai, Jeff PM Lee. The Hong Kong Reference Framework for Hypertension Care for Adults in Primary Care Settings-translating evidence into practice. Hong Kong Pract 2012;34:76-83. 